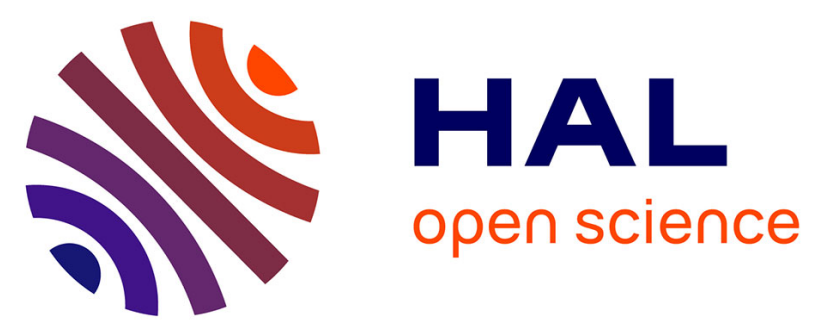

\title{
A Smart-Home IoT Infrastructure for the Support of Independent Living of Older Adults
}

\author{
Stefanos Stavrotheodoros, Nikolaos Kaklanis, Konstantinos Votis, Dimitrios
}

Tzovaras

\section{- To cite this version:}

Stefanos Stavrotheodoros, Nikolaos Kaklanis, Konstantinos Votis, Dimitrios Tzovaras. A Smart-Home IoT Infrastructure for the Support of Independent Living of Older Adults. 14th IFIP International Conference on Artificial Intelligence Applications and Innovations (AIAI), May 2018, Rhodes, Greece. pp.238-249, 10.1007/978-3-319-92016-0_22 . hal-01821303

\section{HAL Id: hal-01821303 \\ https://hal.inria.fr/hal-01821303}

Submitted on 22 Jun 2018

HAL is a multi-disciplinary open access archive for the deposit and dissemination of scientific research documents, whether they are published or not. The documents may come from teaching and research institutions in France or abroad, or from public or private research centers.
L'archive ouverte pluridisciplinaire HAL, est destinée au dépôt et à la diffusion de documents scientifiques de niveau recherche, publiés ou non, émanant des établissements d'enseignement et de recherche français ou étrangers, des laboratoires publics ou privés. 


\title{
A smart-home IoT infrastructure for the support of independent living of older adults
}

\author{
Stefanos Stavrotheodoros ${ }^{1}$, Nikolaos Kaklanis ${ }^{1}$, Konstantinos Votis ${ }^{1}$ and Dimitrios \\ Tzovaras $^{1}$ \\ ${ }^{1}$ Information Technologies Institute, Centre for Research and Technology Hellas, Thessaloniki, \\ Greece \\ \{stavrotheodoros, nkak, kvotis, tzovaras\}@iti.gr
}

\begin{abstract}
Although the healthcare sector has been hugely benefited from the advantages made in the Information and Communication Technology (ICT) domain in the recent years, the emerging technology breakthrough of the Internet-of-Things (IoT), in which all devices and services are collaborating while reducing human intervention, promises new solutions that will enable users to have a more home-centric healthcare, and a sustainable active and healthy ageing. This paper is proposing a smart-home IoT infrastructure for the support and extension of the independent living of older adults in their living environments that responds also to real needs of caregivers and public authorities. The proposed infrastructure seamlessly utilizes health and monitoring devices for the provision of a safe environment for an elderly, the mitigation of frailty and the preservation of quality of life and autonomy. It also provides a mechanism for easy setup and testing of the installed equipment and a decision support system that offers advanced data analytics and visual analytics mechanisms to the formal and informal caregivers of the elderly for the efficient monitoring of their health and activity status.
\end{abstract}

Keywords: Telemedicine, Internet of Things, Elderly monitoring.

\section{Introduction}

Most of the countries are encountering a shift in the distribution of their population towards older ages, caused by rising life expectancy, declining birth rates, or a combination of both.

Although the current health caring model is hospital-centric, it is expected to gradually be transformed to a more hospital-home-balanced by 2020 [1], and eventually to home-centric. Ageing in place does not only reflects the preferences of the elderly who wish to stay in their own homes as they age [2], yet in addition of the policymakers, who are attracted by the possibility of keeping the health care expenditures within the bounds of economic possibility [3].

The effort made in this health caring model transformation is primarily based on the use Ambient Assisted Living (AAL) solutions. Typically, AAL refers to the use of ICT, assistive devices and smart home technologies in a person's daily living to enable individuals living in their place in a convenient and safe manner. It ensures greater 
autonomy and provides supportive home environments by means of integrating sensors, actuators, smart interfaces, and artificial intelligence [4].

IoT emerged as one of the major communication advantages in recent years and is defined as the ability of everyday life objects to connect and communicate with each other [5]. These devices, objects or things are identifiable, readable, recognizable and even controllable via the Internet. With the use of IoT, AAL systems are enhanced and able to consist of medical sensors, wireless sensors, computer hardware, computer networks, software applications, and databases, which can be interconnected to exchange data and provide services in an Ambient Assisted environment [6].

One of the main problems with the IoT is that it is so vast and such a broad concept that there is no proposed, uniform architecture. In order for the idea of IoT to work, it must consist of an assortment of the sensors, network, communications and computing technologies, amongst others [7]. Creating an IoT health system that will ensure the monitoring and independent living of an elderly at his home has many challenges that must be faced. An efficient topology must be selected, and devices produced to different specification and using different communication protocols must be integrated. All vital private information such as personal healthcare information must also be protected by providing confidentiality, integrity, authentication and authorization.

Although several IoT platforms have been developed to support cognitively impaired individuals, a major challenge is to provide a solution that combines efficiently health and behavioural monitoring, thus addressing a large variety of daily needs of the elderly.

This paper presents a smart-home IoT infrastructure for the support of independent living of the elderly developed in the context of the ACTIVAGE H2020 EU project. Specifically, the architecture developed for the Greek deployment site, where a largescale pilot will take place in three different regions with 500 beneficiaries, is presented. The proposed architecture includes a wireless sensor network consisted of devices and sensors of different communication protocols and provides tools for easy installation of the equipment by the personnel. The platform aggregates information gathered by sensors and health devices and integrates them to support the delivery of telehealth services. The collected data are stored in cloud infrastructure and can be accessed only by authorized informal and formal caregivers for monitoring of the health and activity status of the elderly.

The rest of the paper is structured as follows: Section 2 describes several solutions that have been implemented for monitoring and supporting the independent living of elderly people, whereas Section 3 describes the architecture and the layers of the proposed solution along with an in-detail analysis of all used devices/sensors and protocols. Finally, Section 4 concludes the paper.

\section{Related work}

The technological advances in microelectromechanical systems have made available efficient, low cost, low power miniature devices for use in remote monitoring applica- 
tions. Several platforms have been implemented in order to monitor and support the independent living of elderly adults. For example, the IN LIFE system [8] is a cloudbased platform that provides AAL support to cognitively impaired elderly people, through numerous provided tools and services. The MyLife project [9] supports independence for older people with reduced cognitive function by giving them access to simple and intuitive services that adapt to their individual needs and wishes. Although both platforms, meet several needs posed by cognitive decline, they do not utilize the capabilities provided by the IoT infrastructure. On the other hand, numerous solutions adopt the IoT paradigm for the indoor support of elderly people and mainly focus on human activity recognition (HAR) and health monitoring.

The goal of the human activity recognition is to recognize common human activities in real life settings by extracting knowledge from the data acquired by smart sensors [10].

There are HAR solutions that utilize sensors that are placed in various locations within a smart home, acting as non-intrusive monitoring devices for identifying human behaviors. In particular, Kasteren et al. [11] designed a system for recognizing various living activities in a smart home deploying door sensors, pressure-sensitive mats, float sensor, and temperature sensor. For the TAFETA project [12], a homebased automated system has been implemented that monitors the health and wellbeing of an elderly while remaining unobtrusive by using various types of intelligent sensors in the elderly's home. Chen et al. [13] designed a system for recognizing complex living activities in a smart home deploying contact, motion, tilt and pressure sensors, while Zhang et al. [14] also describe a similar system named "Smarter and Safer Home" that deploys sensors in homes, acting as non-intrusive monitoring devices for human behavior of elderly people. The aggregated data of the aforementioned systems are used in order to identify "abnormal" situations.

Other HAR solutions provide activity-monitoring functionalities by using wearable devices for indoor localization. Komai et al. [15] describe an activity monitoring system that utilizes a wearable BLE beacon device for indoor localization. Popleteev [16] presented an activity tracking and indoor positioning system with a wearable magnet and similarly, Belmonte-Fernández et al. [17] by using a Smart-watch wearable device that acquires the Wi-Fi strength signals of surrounding installed Wireless Access Points. Finally, [18] Santos et al., has proposed an RFID based M-health care system using IoT based connected devices, that identifies the position of an m-health related item (e.g. elderly) carrying an RFID tag.

Regarding the health monitoring, its goal is to allow an individual to closely monitor his/her vital signs, provide feedback for maintaining an optimal health status and create alerts when vital measurements are below or over a predefined threshold [19].

Several health-monitoring systems utilize devices that are placed in the living environment of the elderly and are used by him/her periodically during the day for measuring vital signs. Specifically, the system presented by [20] utilizes an electronic blood pressure device that transmits the measured data (i.e., pulse wave) to the data processing center, from where they are accessible in real-time by a doctor. In a similar way, Cao et al. [21] developed an IoT system that measures saturation peripheral oxygen and pulse rate, and transmits them to a cloud-side server. 
Other solutions use wearable bio-signal sensors that monitor human vital signs continuously. Menychtas et al. [22] introduced a mechanism for uniform biosignals collection from wearables and biosignal sensors, and decision support modules for patient monitoring. In [23] a holistic solution for communication and management of Bluetooth biosignal sensors and activity trackers promoting mHealth and self-care is proposed. A glucose monitoring system based on an implantable unit that utilizes Bluetooth low energy, to transmit the measured glucose data to patients' mobile phone or PDA is presented by Ali et al. [24]. Pinto et al. [25] demonstrated a platform for monitoring and registering patients' vital information as well as providing mechanisms to trigger alarms in emergency situations by using a wristband equipped with numerous sensors.

The infrastructure proposed in this paper supports the efficient monitoring of the elderly through a combination of heterogeneous devices that provide both human behavior monitoring and measurement of vital signs of the elderly in his/her living environment in a non-intrusive way. It also supports a mechanism that enables the easy installation and testing of the equipment. The collected data are securely forwarded to a cloud infrastructure offering also decision support functionalities to the formal and informal caregivers of the elderly through advanced data analytics and visual analytics.

\section{Proposed solution}

Before describing the proposed solution, it is essential to present the end-users of the platform and their needs:

- Assisted person: Elderly people, from 65 year and above, with chronic health problems or with frailty due to physical, cognitive or emotional problems, who need formal or informal support when staying at home or in their daily living environment for safety and security.

- Informal caregiver: responsible for providing support on a daily basis to a relative that suffers from a chronic condition and cannot live alone and yet be safe. The service will allow him/her to monitor the relative remotely and respond immediately and effectively in case of an emergency.

- Formal caregiver: responsible for providing systematic daily assistant at home or at a daycare center. The service will allow the formal caregiver to coordinate different care recipients at the same time more efficiently as well as respond to different emergency cases faster.

In order to meet the needs of the aforementioned user groups, an IoT infrastructure was designed and implemented, as it is depicted in Fig. 1, which enables devices with sensing, processing and communication capabilities to be connected to the Internet. It is an extension of the three-layered architecture proposed by [26] where each layer is equivalent to the most basic features of an IoT-based platform: data collection, transmission, and analysis [27]. The installed devices are connected to the Internet through a gateway, forming a Machine-to-Machine (M2M) network. 
A lab-based pilot was used as a reference site to test the proposed infrastructure. Specifically, the smart home of CERTH/ITI was used for living lab testing, providing a fully controllable environment similar to the real cases. The assessment was based on gathering data from different sensors, in which CERTH/ITI employees and a limited number of elderly performed activities of daily living in standardized scenarios. Based on the aforementioned lab-based pilot, the proposed solution produced promising preliminary feedback and results.

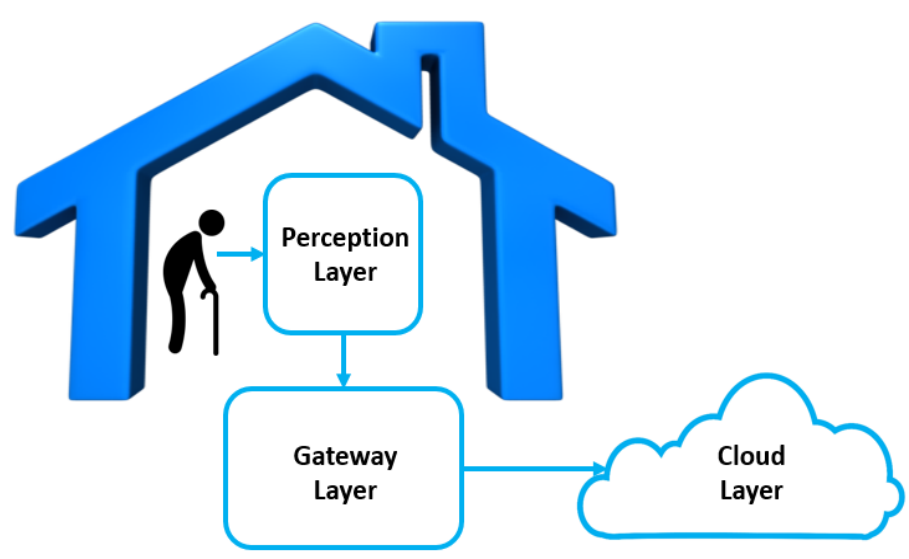

Fig. 1. The adopted three-layered system architecture

\subsection{The Perception Layer}

The first layer that is named Perception Layer (Fig. 2) is responsible for cognizing and collecting information of devices for a user and integrating them into the next layers. It includes a number of heterogeneous, wireless sensors and health devices that create a Wireless Sensor Network (WSN) and a central node is in charge of gathering the sensed data. The central node that is referred as an Aggregation Point in this paper is part of the second layer and is described in detail in the following section. The used devices are using common communication protocols that are featured by low power consumption, short-range communication, and lightweight protocol stack.

The selection of the devices and protocols was made after an in detail examination of the necessities and requirements of the user groups. In particular, these are the protocols used by the selected devices:

- Bluetooth standard [28]

- Z-Wave [29]

- ZigBee [30]

- $\mathrm{RF}(869.2-869.25 \mathrm{MHz})$ 
All devices are battery operated. Consequently, the sensor placement is not constrained by the availability of a nearby power socket, enabling this way an easy installation phase.

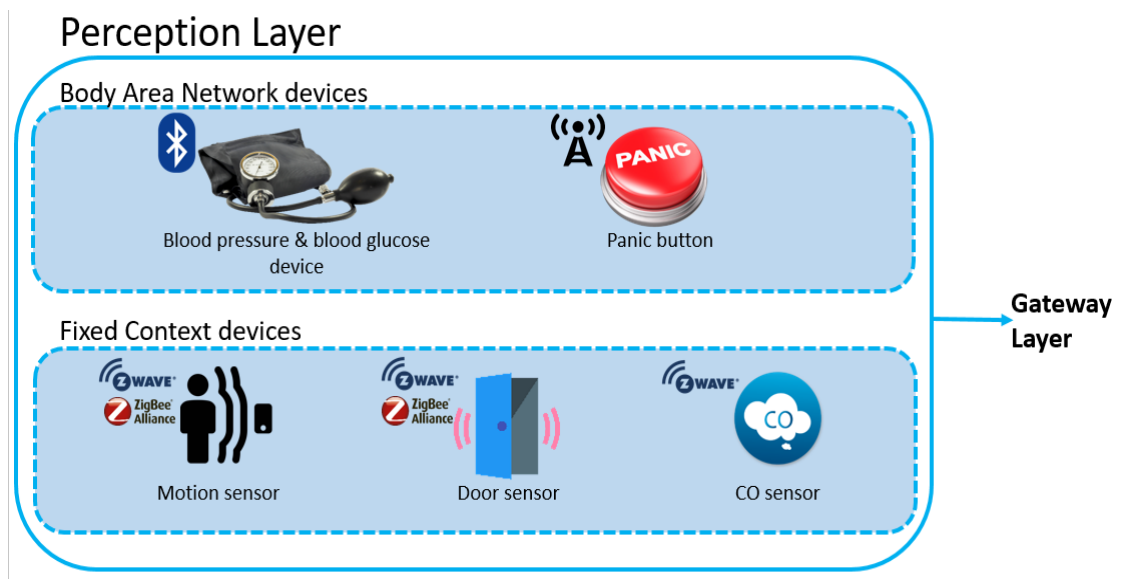

Fig. 2. The Perception Layer that contains all integrated devices

According to [26], the devices in the Perception Layer can be summarized in two main categories: (1) Body Area Network (BAN) devices, which are on-body devices usually for obtaining user health/activity status, and (2) fixed context devices that are generally installed in the home. In a manner corresponding to this categorization, these are the devices used in the implemented system:

- $\quad$ Body Area Network (BAN) devices (transmit on demand)

- Wearable panic button: for emergency cases, a panic button is utilized. It is a small, wearable device, which the elderly has on him. Its activation results in the device automatically transmitting an emergency alarm notification to the carers of the elderly.

- Health device for vital signs: this device is able to measure the vital signs of the elderly, such as the blood pressure and pulses or the blood glucose levels

- $\quad$ Fixed context devices (transmit data on an event basis or as periodic reports)

○ Passive InfraRed (PIR) sensors: by installing Passive Infrared (PIR) sensors in each room, it is possible to track the presence and motion of an elderly in the home and trace occupancy habits.

- Magnetic contact sensors: the central entrance of the home is equipped with a magnetic contact sensor that transmits a signal that indicates the binary state of "open" or "closed".

○ Sensor for measuring hazardous levels of CO: one of the most common symptoms that patients with early stages of Alzheimer's or 
dementia have is the frequent and progressive memory loss [31]. Forgetting to turn off one of the devices that are usually located in the kitchen may turn hazardous for the elderly. By installing this sensor, it is able to determine if there are dangerous levels of $\mathrm{CO}$ in the home.

\subsection{The Gateway Layer}

The second tier is the Gateway layer (Fig. 3), which receives all sensor data and measurements of the perception layer and then transmits them to the third layer. For this layer, a smart device named Aggregation Point is used that undertakes the data aggregator role in the home environment and enables the communication/connection between all installed devices/sensors. It is equipped with a variety of transceiver modules that enable the communication with the devices through the aforementioned protocols, and it features an IoT platform that is responsible for their integration and orchestration. The supported IoT platforms are:

- universAAL [32], which is an open source platform that enables seamless interoperability of devices, services and applications on an unprecedented scale.

- IoTivity [33], which is an open source software framework enabling seamless device-to-device connectivity.

All data collected by the end-devices are forwarded to the Internet, and specifically to the Cloud Layer described in the next section. This is accomplished through an ADSL router or a mobile router $(3 \mathrm{G} / 4 \mathrm{G})$ in cases of homes that do not have an ADSL connection.

The communication protocol used for the transmission of the sensed data to the next layer is the message queuing telemetry transport (MQTT) connectivity protocol and its selection was based on the work produced by Campo et al. [34]. Its simplicity and the fact that it does not need high CPU and memory usage make this protocol ideal for the interconnection between the Gateway Layer and the Cloud Layer. Moreover, it supports an extensive variety of different devices and mobile platforms, and it provides TSL/SSL security at transport layer [35].

Additionally, a mechanism for easy setup and testing of the installed equipment in the smart home by the installer is provided through a web-based application supported by each Aggregation Point. In particular, the aforementioned web-based application supports the configuration of the Aggregation Point for the communication with the Cloud Layer and provides forms for describing and registering the devices/sensors. It communicates with the IoT platform installed on the Aggregation Point via RESTful services exposed for identifying the communication protocol to be used, and handling the pairing and the communication with the devices. The following main functionalities are supported by this application:

- Device registration for adding and describing a new sensor/health device.

- Device removal for removing a sensor/health device from the smart home ecosystem 
- Device discovery by querying the registered devices, along with details regarding their capabilities.

- Device update for updating information related to a device, e.g. change device password.

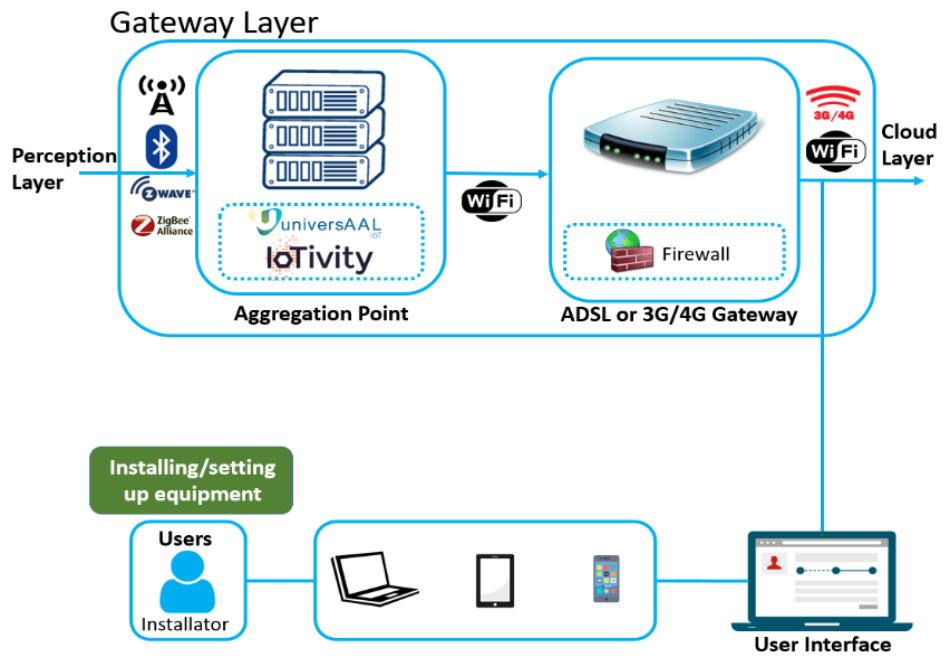

Fig. 3. The Gateway Layer that is responsible for the aggregation of the sensed data

\subsection{The Cloud Layer}

The third layer is the Cloud Layer (Fig. 4) that incorporates a cloud-based software architecture that collects the information gathered from the sensors installed in each home and provides the two following functionalities: (1) storing collected information and (2) analyzing this information and providing a decision support mechanism through advanced data analytics \& visual analytics to the end-users.

For example, the motion sensors are stored in the various rooms of the elderly home and automatically recognize the traceability of each home space. The information transmitted is properly analyzed in order to automatically recognize user habits within a home (e.g. how often he/she visits the bathroom, when he/she visits the kitchen, and for how long, etc.). Recognizing the habits of the elderly contributes to the automatic detection of risk-bearing situations (e.g. the elderly has not visited the bathroom during the day) as well as changes in habits that may be related to health problems (e.g. while the elderly used to be in the kitchen for one hour every noon to prepare his/her daily meal, this custom has changed).

Moreover, the door opening and closing detector that is placed in the central entrance of each home automatically identifies the entrance / exit of the elderly from the house. This information, combined with the information provided by the home-based motion detectors, is properly analysed to identify the user's habits (e.g., how often he/she exits the house, for how long, etc.) and consequently contribute to the automat- 
ic detection of risk situations (e.g. the elderly has not exited home for a long time) as well as changes in habits (for example, a change in the number of the weekly exits and the duration).

The special panic button, which the elderly always has on him, can be used in an emergency case (e.g. fall, severe dizziness, sickness, etc.). At the click of the panic button, the person (s) indicated by the elderly (e.g. relatives, health personnel, etc.) is informed in order to contact with him/her and take appropriate action for help provision. Similarly, an alarm notification will be created in case the blood pressure or blood glucose levels indicate abnormal vital signs.

Regarding the security of the accumulated data, anonymization and encryption techniques are applied. Data anonymization preserves privacy by eliminating identifiability from the dataset, i.e., the link between sensitive information and people, while encryption protects the confidentiality of the data and prevents unauthorized third parties or threat actors from accessing them. Finally, further research is currently made in order to make the platform compliant with the new General Data Protection Regulation (GDPR) [36], which will come into force on May 2018.

The data accumulated is accessible to two different types of users, for whom the following applications have been implemented:

- Application for helping carers (both formal and informal) monitor the health status/activity of the corresponding elderly through advanced data analytics

- Application for offering monitoring and decision support to the administrator of the infrastructure through advanced data analytics.

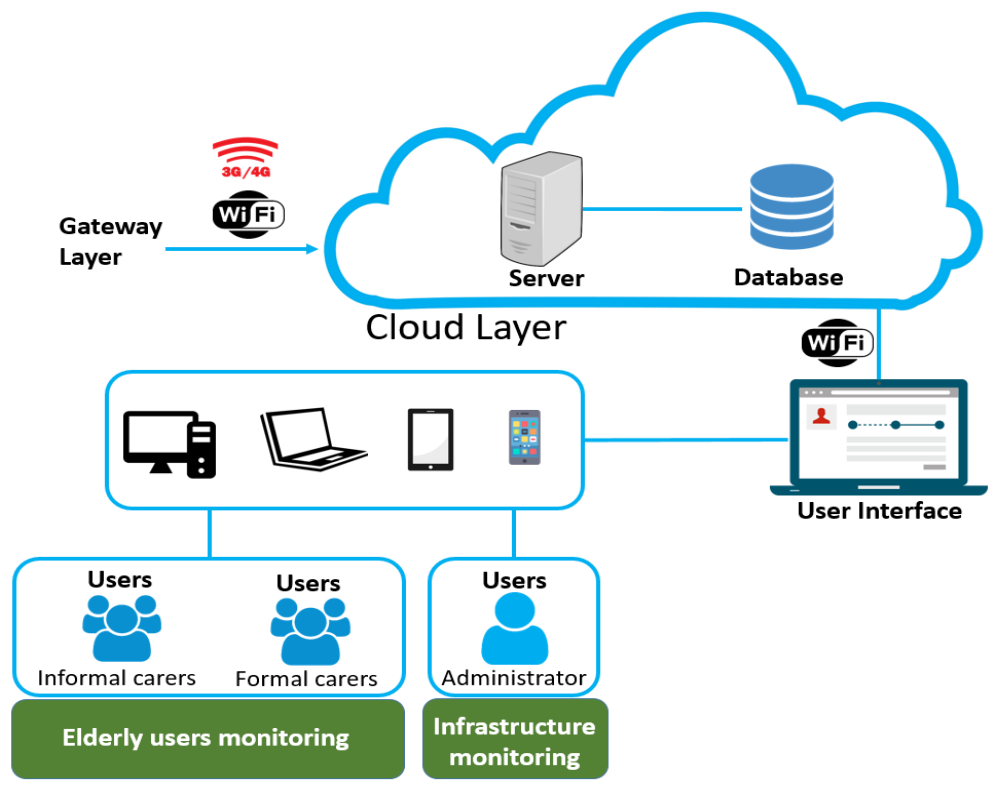

Fig. 4. The Cloud Layer that is responsible for the storing of the information collected from all sites 


\section{Conclusion}

There is a major researching movement that explores various ICT arrangements in order to enhance healthcare provision by mobilizing the potential of IoT. In this paper, we exhibited a smart-home IoT infrastructure created for monitoring the health and activity status of elderly individuals in their living environments. For supporting the independent living of older adults in their living environments and providing perceived safety, a set of heterogeneous sensors, able to provide behavioural and environmental condition information, was utilized in addition to health devices, used for vital sign measurement. The installation and the efficient set up of these devices are feasible through a web-based application implemented for the corresponding personnel. All the aggregated data are collected in a transparent way by the IoT infrastructure deployed in the home and stored securely in a cloud-based infrastructure, where they analysed. The outcome of the analysis is advanced data analytics \& visual analytics presented to the formal and informal caregivers, thus providing them with a decision support mechanism.

The platform will be additionally tested and evaluated through large-scale pilots that will take place in three different regions of Greece, in the context of the ACTIVAGE project. In these pilots, the platform will be deployed in 500 homes and it will provide activity and health status monitoring of elderly people living autonomously in real life scenarios. Inputs from these pilot sites will enable the further evaluation and refinement of the platform.

\section{Acknowledgement}

This work is supported by the EU funded project ACTIVAGE (H2020 - 732679)

\section{References}

1. Koop, C.E., Mosher, R., Kun, L., Geiling, J., Grigg, E., Long, S., Macedonia, C., Merrell, R., Satava, R., Rosen, J.: Future delivery of health care: Cybercare. IEEE Engineering in Medicine and Biology Magazine 27(6), 29-38 (2008).

2. Wiles, J.L.: Home as a new site of health care consumption. In: Andrews, G., Phillips, D.R. (eds.) Aging in Place. Routledge, London (2005).

3. Grabowski, D.: The cost-effectiveness of long-term care services: review and synthesis of the most recent evidence. Medical Care Research and Review 63(1), 3-28 (2006).

4. Sixsmith, A., Mueller, S., Lull, F., Klein, M., Bierhoff, I., Deleaney, S., Byrne, P., Sproll, S., Savage, R., Avatangelou, E.: A user-driven approach to developing ambient assisted living systems for older people: the SOPRANO project. In: Soar, J., Swidell, R., Tsang, P. (eds.) Intelligent Technologies for Bridging the Grey Digital Divide. IGI Global, Hershey PA (2010).

5. Dohr, A., Modre-Opsrian, R., Drobics, M., Hayn, D., Schreier, G.: The Internet of Things for Ambient Assisted Living. In: Proceedings of the Seventh International Conference on Information Technology: New Generations, pp. 804-809. IEEE Press (2010). 
6. Memon, M., Rahr Wagner, S., Pederson Fischer, C., Aysha Beevi, F.H., Overgaard Hansen, F.: Ambient assisted living healthcare frameworks, platforms, standards, and quality attributes. Sensors 14, 4312-4341 (2014).

7. Gigli, M., Koo, S.: Internet of Things, Services and Applications Categorization. Advances in Internet of Things 1, 27-31 (2011).

8. Stavrotheodoros, S., Kaklanis, N., Tzovaras, D.: A Personalized Cloud-Based Platform for AAL Support to Cognitively Impaired Elderly People. In: Maglaveras, N., Chouvarda, I., de Carvalho, P. (eds.) Precision Medicine Powered by pHealth and Connected Health. IFMBE Proceedings 66. pp. 87-91. Springer, Singapore (2018).

9. MyLife project, http://www.mylife-project.org, last accessed 2018/3/19

10. Kim, E., Helal, S., Cook, D.: Human activity recognition and pattern discovery. IEEE Pervasive Computing 9(1), 48-53 (2010).

11. Van Kasteren, T., Englebienne, G., Krose, B. J.: An activity monitoring system for elderly care using generative and discriminative models. Personal and ubiquitous computing, 14(6), 489- 498 (2010).

12. Arcelus, A., Jones, M. H. Goubran, R., Knoefel, F.: Integration of smart home technologies in a health monitoring system for the elderly. In: Proceedings of Advanced Information Networking and Applications Workshops 07. pp 820-825 (2007).

13. Chen, L., Nugent, C. D.: Wang, H.: A knowledge-driven approach to activity recognition in smart homes. Knowledge and Data Engineering, IEEE Transactions 24(6), 961-974 (2012).

14. Zhang, Q., Su, Y., Yu, P.: Assisting an elderly with early dementia using wireless sensors data in smarter safer home. Service Science and Knowledge Innovation, 398-404 (2014).

15. Komai, K., Fujimoto, M., Arakawa, Y., Suwa, H., Kashimoto, Y., Yasumoto, K.: Beaconbased multi-person activity monitoring system for day care center. In: 2016 IEEE International Conference on Pervasive Computing and Communication Workshops (PerCom Workshops), pp. 1-6 (2016).

16. Popleteev, A.: Activity tracking and indoor positioning with a wearable magnet. In: Proceedings of the 2015 ACM International Joint Conference on Pervasive and Ubiquitous Computing and Proceedings of the 2015 ACM International Symposium on Wearable Computers, pp. 253-256 (2015)

17. Belmonte-Fernández, Ó., Puertas-Cabedo, A., Torres-Sospedra, J., Montoliu-Colás, R., Trilles-Oliver, S.: An Indoor Positioning System Based on Wearables for AmbientAssisted Living. Sensors 17(1), 36 (2016).

18. Santos, A., Macedo, J., Costa, A., Nicolau, M.J.: Internet of things and smart objects for M-health monitoring and control. Procedia Technol 16, 1351-1360 (2014)

19. Yap, J. H., Jeong, D. U.: Design and implementation of ubiquitous ECG monitoring system by using android tablet. In: Ubiquitous Information Technologies and Applications 214 of Lecture Notes in Electrical Engineering, pp. 269-277, Springer, Berlin, Germany, (2013).

20. Wang, J., Sun, S., Zhang, K., Zhang, L., Xing, B., Gao, Z.: Smart Blood Pressure Monitoring System Based on Internet of Things. In: CHI 13, (2013).

21. Cao, G., Liu, J.: An IoT Application: Health Care System with Android Devices. In: Gervasi, O. et al. (eds.) Computational Science and Its Applications - ICCSA 2016. ICCSA 2016. Lecture Notes in Computer Science, vol. 9786. Springer, Cham (2016)

22. Menychtas, A., Tsanakas, P., Maglogiannis, I.: Automated integration of wireless biosignal collection devices for patient-centred decision-making in point-of-care systems. Healthcare technology letters 3.1, 34-40 (2016). 
23. Menychtas, A., Padimatos, D., Tsanakas, P., Maglogiannis, I.: On the integration of wearable sensors in IoT enabled mHealth and quantified-self applications. In: Auer, M., Tsiatsos, T. (eds.) Interactive Mobile Communication Technologies and Learning. Advances in Intelligent Systems and Computing 725. Springer, Cham (2017).

24. Ali, M., Albasha, L., Al-Nashash, H.: A bluetooth low energy implantable glucose monitoring system. In: Microwave Conference (EuMC), 2011 41st European. IEEE, pp. 12651268 (2011).

25. Pinto, S., Cabral, J., Gomes, T.: We-care: An IoT-based health care system for elderly people. In: 2017 IEEE International Conference on Industrial Technology (2017).

26. Azimi I., Rahmani A.M., Liljeberg P., Tenhunen H.: Internet of things for remote elderly monitoring: a study from user-centered perspective. Journal of Ambient Intelligence and Humanized Computing 8 (2), 273-289 (2017).

27. Touati, F., Tabish, R.: u-Healthcare system: state-of-the-art review and challenges. Journal of Medical Systems 37(3), 1-20 (2013).

28. Bluetooth Standard, https://www.bluetooth.com/, last accessed 2018/3/19

29. ZWave Alliance, http://www.Z-wave.com, last accessed 2018/3/19

30. ZigBee Alliance, http://www.zigbee.org/, last accessed 2018/3/19

31. Small, G.W.: What we need to know about age related memory loss. BMJ 324, 1502-1505 (2002).

32. Hanke, S., Mayer, C., Hoeftberger, O., Boos, H., Wichert, R., Tazari, M.-R., Wolf, P., Furfari, F.: universAAL - An Open and Consolidated AAL Platform. In: Wichert, R., Eberhard, B. (eds.) Ambient Assisted Living. Advanced Technologies and Societal Change, vol. 63, pp. 127-140. Springer, Heidelberg (2011).

33. Subash, A.: IoTivity - Connecting Things in IoT. In: TIZEN Developer Summit, pp. 1-48 (2015).

34. Campo, A. D., Gambi, E., Montanini, L., Perla, D., Raffaeli, L., Spisante, S.: MQTT in AAL systems for home monitoring of people with dementia. In: Proceedings of the 27th Annual IEEE International Symposium on Personal, Indoor and Mobile Radio Communications (PIMRC), pp. 1-6 (2016).

35. Asim, M.: A Survey on Application Layer Protocols for Internet of Things (IoT). International Journal of Advanced Research in Computer Science 8(3), 996-1000 (2017).

36. General Data Protection Regulation (GDPR), https://www.eugdpr.org/, last accessed $2018 / 3 / 19$ 